\title{
NOTE ON THE INEQUALITIES OF J. KAZDAN ${ }^{1}$
}

\author{
WILL Y. LEE \\ Rutgers University - Camden \\ Department of Mathematics \\ Camden, NJ 08102, USA
}

\begin{abstract}
In this note, we prove the Kazdan's inequalities without using what is called the Heisenberg uncertainty principle. Instead we prove it using Garofalo-Lin inequality among other things.

Key words: Heisenberg uncertainty principle, unique continuation theorem, Garofalo-Lin inequality, Schwarz inequality, Poincare inequality. 49.

AMS (MOS) subject classifications: Primary: 35, Secondary:
\end{abstract}

\section{INTRODUCTION}

In [4], J. Kazdan has shown strong unique continuation theorem (Theorem 1.8 of [4]) whose proof is mainly based on his main lemma (Lemma 2.4 of [4]). Several analytic as well as geometric inequalities were used to prove the main lemma. Among them are the following inequalities:

There exist constants $C_{1}, C_{2}, C_{3}, C_{4}, C_{5}$ and $r_{0}$ such that for all $r \in\left(0, r_{0}\right)$

$$
\begin{aligned}
& \left|I_{j}(r)\right| \leq C_{j} f(r)(H(r)+D(r))(j=1,2) \\
& \frac{1}{r^{n-2}} \int_{\partial B_{r}}|\nabla u|^{2} d S \leq r B(r)+C_{3} H(r)+D(r) \\
& \mid I_{3}(r) \leq C_{4} f(r)(H(r)+D(r)+\sqrt{r H(r) B(r)}) \\
& \left|I_{4}(r)\right| \leq C_{5} f(r)(H(r)+D(r)+r B(r)) .
\end{aligned}
$$

\footnotetext{
${ }^{1}$ Received: February, 1992. Revised: July, 1992.
} 
Here $f(r), I_{1}(r), I_{2}, I_{3}(r), H(r), D(r), B(r)$ are defined as follows: let $f$ be a smooth increasing function with $f(0)=0$ satisfying $\int_{0}^{r_{0}} \frac{f(r)}{r} d r<\infty$ and let $u$ satisfy for $n \geq 3$ the differential inequality with $a$ and $b$ constants:

$$
\begin{aligned}
& |\Delta u(x)| \leq \frac{a f(r)}{r^{2}}|u(x)|+\frac{b f(r)}{r}|\nabla u(x)| \\
& I_{1}(r)=\frac{1}{r^{n-2}} \int_{B_{r}} u \Delta u d V \\
& I_{2}(r)=\frac{2}{r^{n-2}} \int_{B_{r}} \rho u_{\rho} \Delta u d V \\
& I_{3}(r)=\frac{1}{r^{n-3}} \int_{\partial B_{r}} u \Delta u d S \\
& H(r)=\frac{1}{r^{n-1}} \int_{\partial B_{r}}|u|^{2} d S \\
& D(r)=\frac{1}{r^{n-2}} \int_{B_{r}}|\nabla u|^{2} d V \\
& B(r)=\frac{2}{r^{n-2}} \int_{\partial B_{r}} u_{\rho}^{2} d S .
\end{aligned}
$$

In his proof of inequalities $(1),-(2)$, Kazdan relies on what is called the Heisenberg uncertainty principle (see [2], [3], \& [4]):

$$
\begin{aligned}
& \int_{B_{r}} \frac{w^{2}}{\rho^{2}} d V \leq \frac{c}{r} \int_{\partial B_{r}} w^{2} d S+\widetilde{C} \int_{B_{r}}|\nabla w|^{2} d V, n \geq 3 \\
& \int_{B_{r}} \frac{2|w||\nabla w|}{\rho} d V \leq \frac{C^{\prime}}{r} \int_{\partial B_{r}} w^{2} d S+\tilde{C}^{\prime} \int_{B_{r}}|\nabla w|^{2} d V, n \geq 3
\end{aligned}
$$

where $C$ and $\widetilde{C}$ are dimensional constants. Inequality (13) is an easy consequence of (12). Indeed a straightforward computation shows that $C^{\prime}=\frac{C \lambda}{r}, \widetilde{C}^{\prime}=\lambda\left(\frac{1}{\lambda^{2}}+\widetilde{C}\right)$ for any $\lambda>0$. 
Since there is nothing to comment on the proofs of inequalities (3) and (4), we prove inequalities $(1)_{j}$ and (2) without using the Heisenberg uncertainty principle (12)-(13). Instead we use the following lemma which is the Garofalo-Lin inequality (see 4.11 of [2]) applied to the operator $L$ where

$$
L u=-\Delta u+b(x) \cdot \nabla u+V(x) u=0 .
$$

Here $b(x)$ and $V$ are majorized by with constants $a$ and $b$ :

$$
|b(x)| \leq \frac{b f(r)}{r},|V(x)| \leq \frac{a f(r)}{r^{2}}
$$

Lemma: Let $u \in w_{\text {loc }}^{1,2}$ satisfy equation (14). Then there exists a small constant $r_{0} \in(0,1)$ depending on $n, b, V$ and $u$ such that for all $r \in\left(0, r_{0}\right)$

$$
r \int_{\partial B_{r}} u^{2} d S \geq \int_{B_{r}} u^{2} d V
$$

Proof: First observe that

$$
\begin{aligned}
& \int_{B_{r}} u(b(x) \cdot u)\left(r^{2}-|x|^{2}\right) d V \\
& \leq \int_{B_{r}}|u||b(x)||\nabla u|\left(r^{2}-|x|^{2}\right) d V \\
& \leq\|b\|_{L^{\infty}}\left(\int_{B_{r}} u^{2}\left(r^{2}-|x|^{2}\right) d V\right)^{1 / 2}\left(\int_{B_{r}}|\nabla u|^{2}\left(r^{2}-|x|^{2}\right) d V\right)^{1 / 2} \\
& \leq\|b\|_{L} \infty r_{0}^{2}\left(\int_{B_{r}} u^{2} d V\right)^{1 / 2}\left(\int_{B_{r}}|\nabla u|^{2} d V\right)^{1 / 2} \\
& \leq C\|b\|_{L^{\infty}} r_{0}^{2}\left(\int_{B_{r}}|\nabla u|^{2} d V\right) \quad \text { (Poincare inequality) }
\end{aligned}
$$

where $C$ is a dimensional constant. Consequently we obtain

$$
\int_{B_{r}} u(b(x) \cdot \nabla u)\left(r^{2}-|x|^{2} d V \geq-C\|b\|_{L^{\infty}} r_{0}^{2} \int_{B_{r}}|\nabla u|^{2} d V .\right.
$$

Choose $r_{0}$ so small that

$$
r_{0}^{2} \leq 1 /\left(C\|b\|_{L^{\infty}} \int_{B_{r}}|\nabla u|^{2} d V \int_{B_{r}} u^{2} d V\right)
$$


Inequalities (17)-(18) then reveal that

$$
\int_{B_{r}} u(b(x) \cdot \nabla u)\left(r^{2}-|x|^{2}\right) d V \geq-\int_{B_{r}} u^{2} d V .
$$

Secondly we have

$$
\int_{B_{r}} V u^{2}\left(r^{2}-|x|^{2}\right) d V \geq-\|V\|_{L^{\infty}} r_{0}^{2} \int_{B_{r}} u^{2} d V
$$

Choose $r_{0}$ such that

$$
r_{0}^{2} \leq(n-2) /\|V\|_{L^{\infty}}
$$

Inequalities (20)-(21) then show that

$$
\int_{B_{r}} V u^{2}\left(r^{2}-|x|^{2}\right) d V \geq-(n-2) \int_{B_{r}} u^{2} d V
$$

Finally integration by parts and equation (14) give us the following identity:

$$
\int_{B_{r}}\left(|\nabla u|^{2}+u b(x) \cdot \nabla u+V u^{2}\right)\left(r^{2}-|x|^{2}\right) d V=r \int_{\partial B_{r}} u^{2} d S-n \int_{B_{r}} u^{2} d V .
$$

Equation (23) combined with inequalities (19) and (22) shows that

$$
\begin{gathered}
r \int_{\partial B_{r}} u^{2} d S \geq \int_{B_{r}}|\nabla u|^{2}\left(r^{2}-|x|^{2}\right) d V-\underset{B_{r}}{\nabla} u^{2} d V-(n-2) \int_{B_{r}} u^{2} d V \\
+n \int_{B_{r}} u^{2} d V \\
\geq-\int_{B_{r}} u^{2} d V-(n-2) \int_{B_{r}} u^{2} d V+n \int_{B_{r}} u^{2} d V \\
=\int_{B_{r}} u^{2} d V
\end{gathered}
$$

for all $r \in\left(0, r_{0}\right)$ where $r_{0}$ is chosen to be the minimum of the right hand sides of inequalities (18) and (21). This completes the proof.

We give the proof of $(I)_{1}$ only as the proofs of $(I)_{2}$ and (2) are essentially the same.

Proof of $(I)_{1}: \quad\left|I_{1}(r)\right| \leq \frac{1}{r^{n-2}} \int_{B_{r}}|u||\Delta u| d V$

$$
\leq \frac{1}{r^{n-2}} \int_{B_{r}}|u|\left(\frac{a f(r)}{r^{2}}|u|+\frac{b f(r)}{r}|\nabla u|\right) d V
$$




$$
\begin{gathered}
\leq \frac{a f(r)}{r^{n-1}} \int_{\partial B_{r}} u^{2} d S+\frac{b f(r)}{r^{n-1}}\left(\int_{B_{r}} u^{2} d V\right)^{1 / 2}\left(\int_{B_{r}}|\nabla u|^{2} d V\right)^{1 / 2} \\
\leq \frac{a f(r)}{r^{n-1}} \int_{\partial B_{r}} u^{2} d S+\frac{b f(r)}{r^{n-1}}\left(\frac{1}{2 r} \int_{B_{r}} u^{2} d V+\frac{r}{2} \int_{B_{r}}|\nabla u|^{2} d V\right) \\
\leq a f(r) H(r)+\frac{b}{2} f(r) H(r)+\frac{b}{2} f(r) D(r) \quad \text { (Lemma and Schwarz) } \\
\leq C_{1} f(r)(H(r)+D(r))
\end{gathered}
$$

where $C_{1}=a+b / 2$. This complete the proof of $(I)_{1}$.

A simple computation shows inequalities $(I)_{2},(2),(3)$ and (4) are satisfied with $C_{2}=a+2 b, C_{3}=(n-2)+(n+2) \gamma+C_{2} f(r), C_{4}=a+(3 b / 2) \sqrt{C_{3}}, C_{5}=(3 / 2) C_{4}$, where $\gamma$ satisfies $0(r)<(n+2) \gamma$.

\section{REFERENCES}

[1] M.S. Baouendi and E.C. Zachmanoglou, "Unique continuation of partial differential equations and inequalities from manifolds of any dimension”, Duke Math. J. 45, (1978), pp. 1-13.

[2] N. Garofalo and F.H. Lin, "Monotonicity properties of variational integrals $A_{p}$ weights and unique continuations", Indiana Univ. Math. J. 35, (1986), pp. 245-268.

[3] , "Unique continuation for elliptic operators: A geometric-variational approach”, Comm. Pure \& Applied Math XL, (1987), pp. 347-366.

[4] J.L. Kazdan, "Unique continuation in geometry", Comm. Pure \& Applied Math. XLI, (1988), pp. 667-681.

[5] D. Gilbarg and N.S. Trudinger, "Elliptic Partial Differential Equations of Second Order", Springer-Verlag, 1983. 


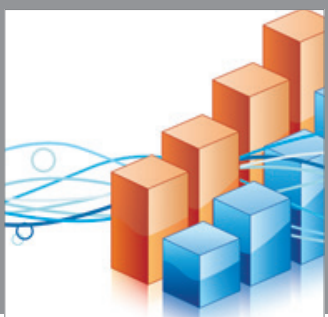

Advances in

Operations Research

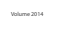

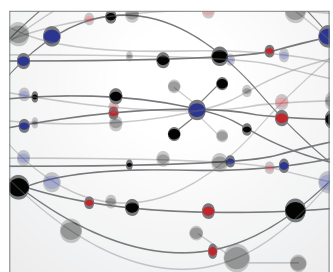

\section{The Scientific} World Journal
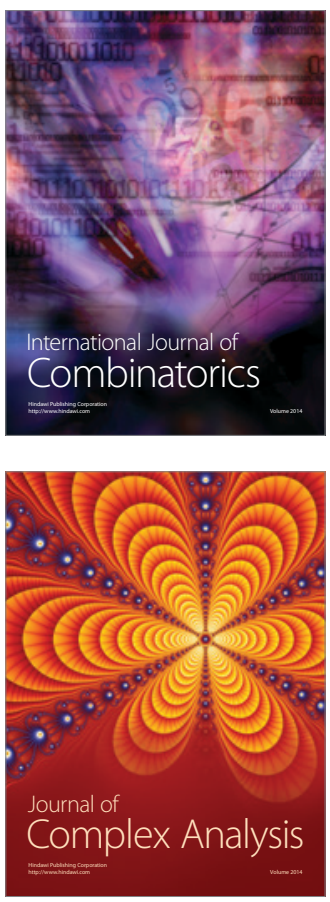

International Journal of

Mathematics and

Mathematical

Sciences
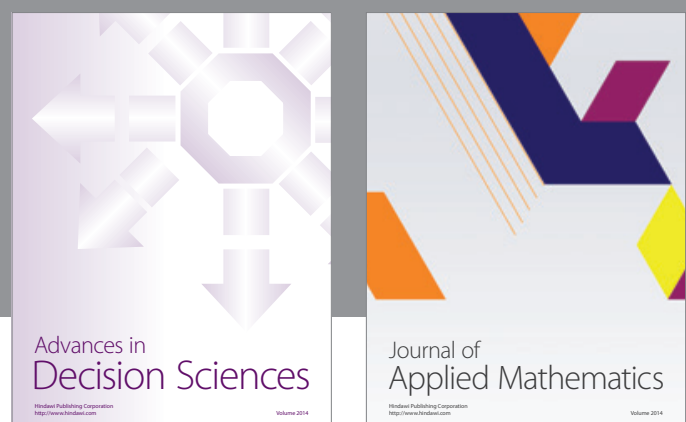

Journal of

Applied Mathematics
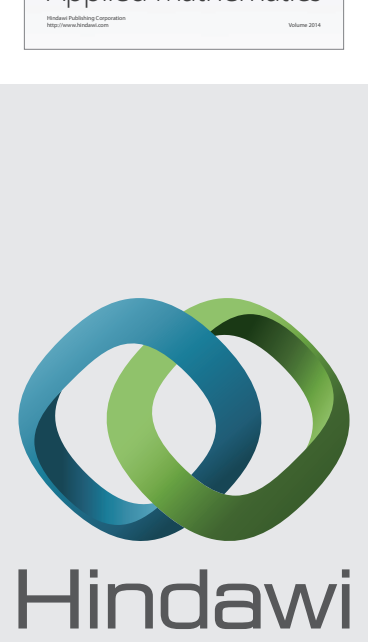

Submit your manuscripts at http://www.hindawi.com
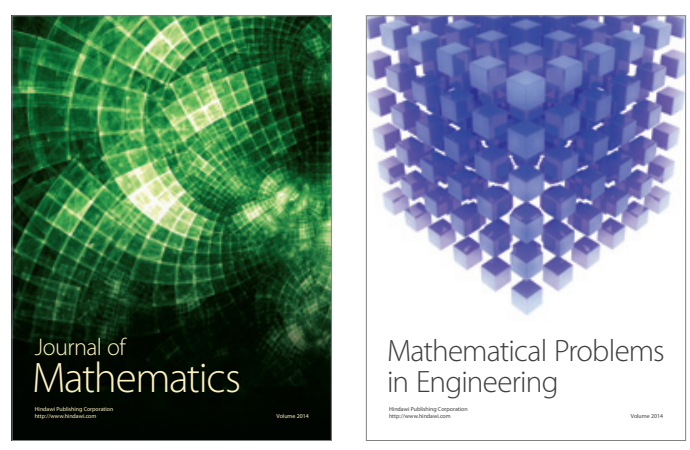

Mathematical Problems in Engineering
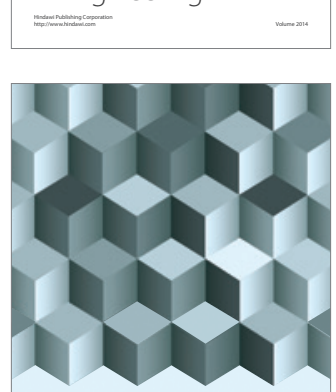

Journal of

Function Spaces
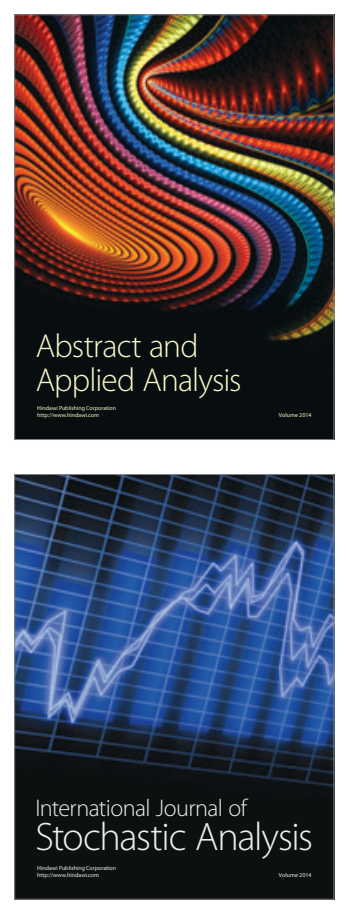

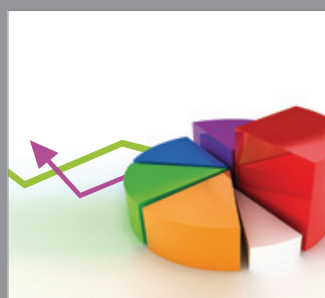

ournal of

Probability and Statistics

Promensencen
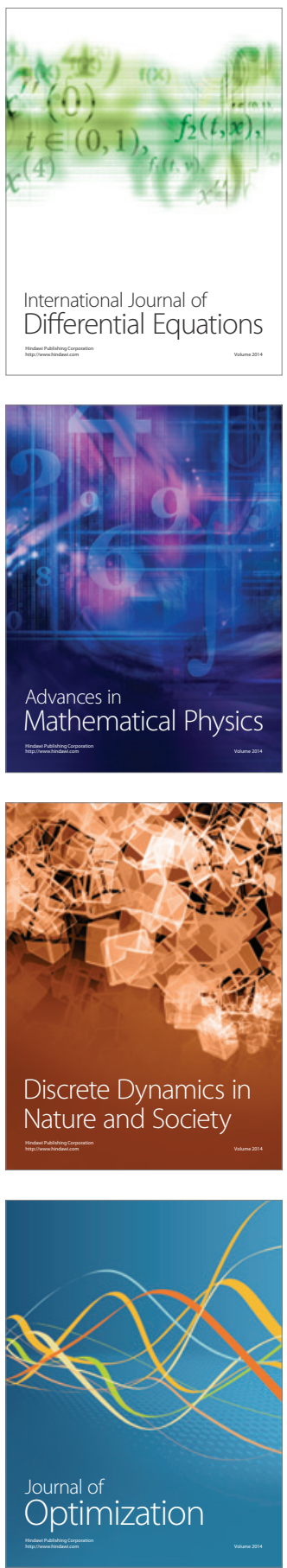